\title{
The use of an XRF glass database to assign a significance to forensic evidence
}

\section{Ruthmara Corzo}

National Institute of Standards and Technology, United States

Glass is an important type of forensic evidence that is frequently encountered in crime scenes. The elemental analysis of glass, using sensitive techniques such as micro-X-ray Fluorescence Spectrometry $(\mu \mathrm{XRF})$ and Laser Ablation-Inductively Coupled Plasma-Mass Spectrometry (LA-ICP-MS), has been shown to be a valuable tool for the discrimination of glass samples originating from different sources and the association of glass samples originating from the same source [1-3]. Although the forensic community has reached a consensus on the analytical protocol for the forensic examination of glass using $\mu$ XRF or LA-ICP-MS $[4,5]$, there is no consensus on how to report the significance of an association or exclusion. To address this gap, recent research efforts have focused on the development of glass databases to explore the use of more objective methods of evidence interpretation (e.g., coincidental match probability, likelihood ratio) and to assign a significance to the evidence. However, these efforts have primarily focused on the development of LA-ICP-MS glass databases, despite the more widespread use of $\mu$ XRF for the elemental analysis of glass fragments in forensic casework [6, 7]. This study focuses on the development of a $\mu$ XRF glass database that can potentially be used by practitioners to assign a weight to forensic glass evidence. The database includes 420 panes of soda-lime float glass, the type of glass that is most commonly encountered in casework, from automotive windshields. Typically, a semi-quantitative approach (using $\mu \mathrm{XRF}$ element ratios) is used to compare the chemical composition of the known and questioned specimens submitted for casework analysis. However, a quantitative approach would allow the $\mu \mathrm{XRF}$ glass database to be shared among practitioners. Additionally, a quantitative $\mu \mathrm{XRF}$ glass database can potentially be combined with existing quantitative LA-ICP-MS glass databases to generate larger collection sets. Therefore, the well-known fundamental parameters method will be applied to quantify the chemical composition of the 420 glass samples using $\mu$ XRF. The quantitative $\mu$ XRF results will be validated using LA-ICP-MS measurements. The application of more objective methods to evidence interpretation using a quantitative $\mu \mathrm{XRF}$ glass database and the agreement between $\mu \mathrm{XRF}$ and LA-ICP-MS will be discussed.

\section{References}

1. T. Trejos, et. al, Cross-validation and evaluation of the performance of methods for the elemental analysis of forensic glass by $\mu$-XRF, ICP-MS, and LA-ICP-MS, Analytical and Bioanalytical Chemistry 405 (16), 2013, 5393-409.

2. T. Trejos, et. al, Forensic analysis of glass by $\mu$-XRF, SN-ICP-MS, LA-ICP-MS and LA-ICPOES: evaluation of the performance of different criteria for comparing elemental composition, Journal of Analytical Atomic Spectrometry 28 (8), 2013, 1270-1282.

3. R. Corzo, et. al, An Interlaboratory Study Evaluating the Interpretation of Forensic Glass Evidence Using Refractive Index Measurements and Elemental Composition, Forensic Chemistry, accepted 2021

4. $\quad$ ASTM Standard E2926-17 Standard Test Method for Forensic Comparison of Glass Using Micro X-ray Fluorescence ( $\mu$-XRF) Spectrometry, ASTM International

5. ASTM Standard E2927-16e1 Standard Test Method for Determination of Trace Elements in SodaLime Glass Samples Using Laser Ablation Inductively Coupled Plasma Mass Spectrometry for Forensic Comparisons, ASTM International

6. R. Corzo, et. al, The Use of LA-ICP-MS Databases to Estimate Likelihood Ratios for the Forensic Analysis of Glass Evidence, Talanta 186 2018, 655-661. 
7. T. Hoffman, et al., An Inter-Laboratory Evaluation of LA-ICP-MS Analysis of Glass and the Use of a Database for the Interpretation of Glass Evidence, Forensic Chemistry 11 2018, 65-76. 